\title{
Constraining Secluded Dark Matter models with the ANTARES neutrino telescope
}

\author{
Miguel Ardid \\ IGIC - Universitat Politècnica de València \\ Paranimf 1, E-46730 Gandia, Spain \\ E-mail: mardidefis.upv.es
}

Christoph Tönnis ${ }^{1}$, on behalf of the ANTARES Collaboration

IFIC - Instituto de Física Corpuscular, CSIC - Universitat de València

Edificios Investigación de Paterna, Apdo. de Correos 22085, E-46071 Valencia, Spain

E-mail: ctoenniseific.uv.es

In this work we describe the search for Secluded Dark Matter (SDM) annihilation in the Sun with ANTARES. SDM is a special scenario where DM, which would gravitationally accumulate in astrophysical objects like the Sun, is annihilated into a pair of non-Standard Model mediators, which subsequently decay into SM particles. It was suggested to explain some experimental observations, such as the positron-electron ratio observed by satellite detectors. Three different cases are studied: a) direct detection of di-muons from the mediator decay, or neutrino detection from: b) the mediator that decays into di-muons and, in turn, into neutrinos, and c) the mediator that directly decays into neutrinos. The ANTARES results obtained for SDM models -the first experimental limits established directly in neutrino telescopes- are presented. The limits imposed to these models are much more restrictive than those derived in direct detection searches for the case of spin-dependent interaction for a wide range of lifetimes of the metastable mediator.

The 34th International Cosmic Ray Conference

30 July- 6 August, 2015

The Hague, The Netherlands

${ }^{1}$ Presenter 


\section{Introduction}

In this paper we present the results of the analysis of ANTARES data in order to search for signatures of Secluded Dark Matter (SDM) annihilation in the Sun. There is strong cosmological and astrophysical evidence about the existence of Dark Matter (DM) in the Universe. There is as well a large consensus that this kind of matter, about $83 \%$ of the total, has the properties of being non-baryonic, non-relativistic and inert to electromagnetic interactions, being the Weakly Interacting Massive Particles (WIMPs) hypothesis the favourite scenario for the nature of DM. Then, DM would be embedded in the visible baryonic part of galaxies forming a halo. In the most common scenario, WIMPs can scatter elastically with matter and become trapped in massive astrophysical objects like the Sun. There, DM particles could selfannihilate reaching a balance between capture and annihilation rates over the age of the Solar System. Usually, the products of DM annihilation are Standard Model (SM) particles, which interact with the interior of the Sun and are largely absorbed. However, during this process, high-energy neutrinos may be produced, which could scape and can be observed by neutrino detectors, such as ANTARES. In this sense, limits on WIMP DM annihilation in the Sun have been reported already in ANTARES [1], and in other neutrino telescopes: Baksan [2], SuperKamiokande [3] and IceCube [4]. Another hypothesis is based on the idea that DM will be Secluded from SM particles, being the annihilation only possible through a metastable mediator $(\phi)$, which subsequently decays into SM states[5-9]. In all these models, the thermal relic WIMP DM scenario is considered as usual while there is also the potential to explain some astrophysical observations, such as the positron-electron ratio observed by PAMELA [10] or FERMI [11], measured recently by AMS-II with much more accuracy [12]. In the Secluded Dark Matter scenario, the presence of a mediator, as a communication way between DM and SM, can dramatically change the annihilation signature of DM captured in the Sun. If the mediators live long enough to escape the Sun before decaying, they can produce detectable charged-particle, $\gamma$-ray or neutrinos $[13,14]$ that could reach the Earth and be detected. In many of the secluded dark matter models, $\phi$ can decay into leptons near the Earth. Some differences appear in the signature of leptons created by the neutrino interaction and leptons arising from $\phi$ decays. In the latter case as the DM mass $(\sim 1 \mathrm{TeV})$ is greater than the $\phi$ mass $(\sim 1 \mathrm{GeV})$ the leptons may be boosted and parallel. If these leptons are muons the signature in the vicinity of the detector would be two muon tracks almost parallel. Meade et al. [15] discuss this possibility and calculate the expected sensitivity for the Icecube neutrino telescope to these cases. It is worth also to mention that even in the case that the di-muon signature could be interpreted as a single muon, the different energy deposition can help to better discriminate this case from the atmospheric neutrino signal [16]. Even for short-lived mediators that decay before reaching the Earth, neutrinos from the products of mediator decays could be detected in neutrino telescopes. Another possibility is that mediators may decay directly into neutrinos, as discussed by [17]. In this case, the neutrino signal could be enhanced significantly compared to the standard scenario even for quite short-lived mediators, since they will be able to escape the dense core of the Sun where high energy neutrinos can interact with nuclei and be absorbed. The fact that the solar density decreases exponentially with radius facilitates that the neutrinos injected by mediators at larger radii propagate out of the Sun because they undergo much less absorption. 
In this work an indirect search for SDM using the 2007-2012 data recorded by the ANTARES neutrino telescope is reported by looking at the different mediator decay products: a) direct detection of di-muons b) neutrinos from decays of di-muons produced by mediators that decay before reaching the Earth and c) neutrinos produced by mediators that decay directly to neutrinos and antineutrinos. The analysis procedure is basically the same as the previous search for dark matter annihilation in the Sun [1], but optimizing the search for the expected signal in the case of SDM.

\section{The ANTARES neutrino telescope}

A description of the ANTARES neutrino telescope can be found elsewhere in these proceedings. A more detailed description of the telescope, subsystems and methods can be found in [18-21]. In this analysis, data recorded between the 27th of January 2007 and the 31st of October 2012 are used, corresponding to a total lifetime of 1321 days, without taking into account the visibility of the Sun. During this time, the detector consisted of 5 lines for most of 2007 and of successively 8, 9, 10 and 12 lines from 2008 to 2012.

\section{Signal and Background estimation}

Two main sources of background are present in ANTARES: 1) Down-going atmospheric muons resulting from the interaction of cosmic rays in the atmosphere. These background events are strongly reduced by the deep sea location and by the reconstruction algorithms that are tuned to up-going events. Cuts on the quality of the tracks are also applied to reject downgoing muons wrongly reconstructed as up-going. 2) Atmospheric neutrinos produced by cosmic rays. These neutrinos can traverse the Earth, so they can be detected as upgoing tracks. This is an irreducible background. Both kinds of background have been simulated and good agreement with data has been found [1]. However, the background estimation is done using scrambled data, by randomizing the time of selected events, to reduce the effect of systematic uncertainties (efficiency of the detector, assumed atmospheric fluxes, etc.).

Regarding to the signal estimation and to be able to evaluate SDM models, a new tool for Di-Muon signal generation (DiMugen) has been developed to evaluate the sensitivity of ANTARES to the the case a) where dimuons are detected directly [22]. DiMugen generates and propagates dimuons coming from decay of mediators resulting from dark matter annihilation. For this analysis, the mediator arrives from the Sun's direction following the zenith and azimuth information about the Sun position during the period under study. Different DM masses in the range between $30 \mathrm{GeV}$ to $10 \mathrm{TeV}$ have been simulated using in most cases a typical mass of 1 $\mathrm{GeV}$ for the mediator $\phi$. Once the muons are generated in the vicinity of the detector according to these conditions, simulations of the travel and interactions of muons are made, as well as the detection of the Cherenkov light by the optical modules. Triggering and reconstruction algorithms are also included in the process in order to evaluate the global efficiency for the detection of dimuons as a function of the quality parameter, $Q$, and the angular deviation from the Sun direction observed, $\Psi$.

To determine the ANTARES sensitivity for the cases where the neutrino is the final decay product that arrives to the Earth, we have used the ANTARES effective areas for neutrinos as functions of the Q and $\Psi$ according to neutrino (and antineutrino) simulations. For this, it is necessary to know the energy spectra of neutrinos arriving to the detector. In case b) the 
neutrino spectra have been obtained from Michel's spectra of neutrinos and antineutrinos from muon decay and taking into account the boost. For scenario c) and assuming long lifetime mediators with respect to the time required to go out from Sun's core, the neutrino (and antineutrino) spectra are almost flat in the energy region under study [17]. For these cases, a detailed neutrino oscillation study has not been done, but the conservative assumption that after oscillations all neutrino flavours arrive to the Earth with the same ratio 1:1:1 has been made.

\section{Optimization of the event selection criteria}

In order to avoid any bias in the event selection, a blinding policy has been followed. The values of the cuts have been chosen before looking at the region where the signal is expected. The best sensitivities for di-muon flux and cross-sections are extracted with the Model Rejection Factor (MRF) method [23]. It consists in finding the set of cuts which provide, in average, the best flux upper limit taking into account the existing background and the efficiency to a possible flux signal from simulations. MRF is used to optimize the half-cone angle around the sun $(\Psi)$ and the track quality cut parameters $(\mathrm{Q})$ for the different cases and the different DM masses studied. Finally, since in most of the cases the difference in flux sensitivities between different optimisations were not large, it was decided to limit the optimisations to 4 different cuts that were representative enough of all possible situations. There are 3 optimisations corresponding roughly to lower, intermediate and larger DM masses for the dimuon detection case. For the neutrino detection cases, the latter one is also used for larger DM masses and another additional optimisation is used for lower and intermediate DM masses.

\section{Results and discussion}

After the optimisation of the flux sensitivities using the MRF with scrambled data, we have looked at the data coming from the Sun's direction. As an example, Figure 1-left shows the distribution of events detected by ANTARES for $\mathrm{Q}<1.8$ as a function of the angle deviation from the Sun. Good agreement between data and the expected background obtained from scrambled data is observed. The green line shows the angle cut selected for this analysis. Since no significant excess is observed in any of the blind cuts proposed, the 90\% CL upper limit values in the Feldman-Cousins approach [24] have been extracted and used to constrain the models. The resulting flux limits for the different cases studied are shown in Figure 1-right.

Following the reasoning given in ref. [15], the di-muon (or neutrino) flux at Earth can be translated into DM annihilation in the Sun through the channel $\mathrm{DM}+\mathrm{DM}->\phi+\phi->(2 \mu)+(2 \mu)$, considering the muon decays for the detection of neutrinos. For the case in which mediators decay directly into neutrinos, only the situation in which the mediator life is long enough has been considered, so that the absorption of neutrinos in the Sun becomes negligible. In this case, the neutrino spectrum is harder and the signal in a neutrino telescope is enhanced. If the lifetime of the mediator is small, the final situation would be quite similar to the typical hard spectrum channels [17]. The conservative assumption that after oscillations all neutrino flavours arrive to the Earth with the same ratio 1:1:1 has been made. Assuming $100 \%$ branching ratios, and taking into account the solid angle suppression and the decay probabilities, as explained in ref. [22], we can start to constrain the models by means of exclusion plots for the annihilation rates as a function of mediator lifetime and dark matter mass. For example, figure 3 shows the ANTARES exclusion limits for the Secluded DM 
scenarios studies for DM masses of 0.5 and $5 \mathrm{TeV}$ using a typical $\phi$ mass of $1 \mathrm{GeV}$. Blue lines indicate the exclusion region in the di-muon case, either by direct detection (dot-dashed line) or through detection of neutrinos (solid line). For large decay length $L=\gamma c \tau,(\mathrm{L}>1 \mathrm{AU})$, that is long mediator lifetime, the direct detection of di-muons is more efficient than neutrino detection for small DM masses, whereas the opposite holds for larger masses. The transition is around 0.8 $\mathrm{TeV}$ in DM mass. Naturally, for small $\mathrm{L}, \mathrm{L}<<1 \mathrm{AU}$, neutrino detection is much more efficient for all DM masses. Green lines indicate the exclusion regions of secluded DM into neutrinos. More stringent constrains are obtained in this scenario, mainly due to the harder neutrino energy spectrum.
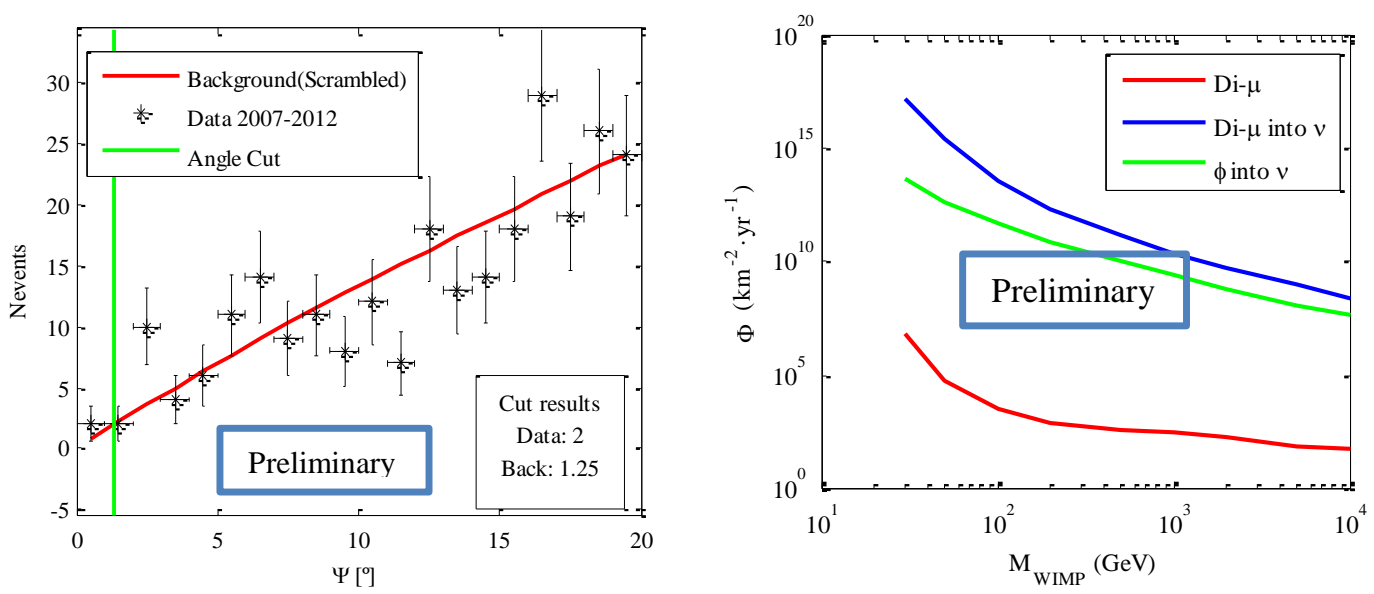

Figure 1. Left: differential distribution of the angular separation of the event tracks with respect to the Sun's direction with $\mathrm{Q}<1.8$ for data (black) and expected background (red line). Right: Limits for the flux of di-muons and neutrinos from the SDM cases studied.
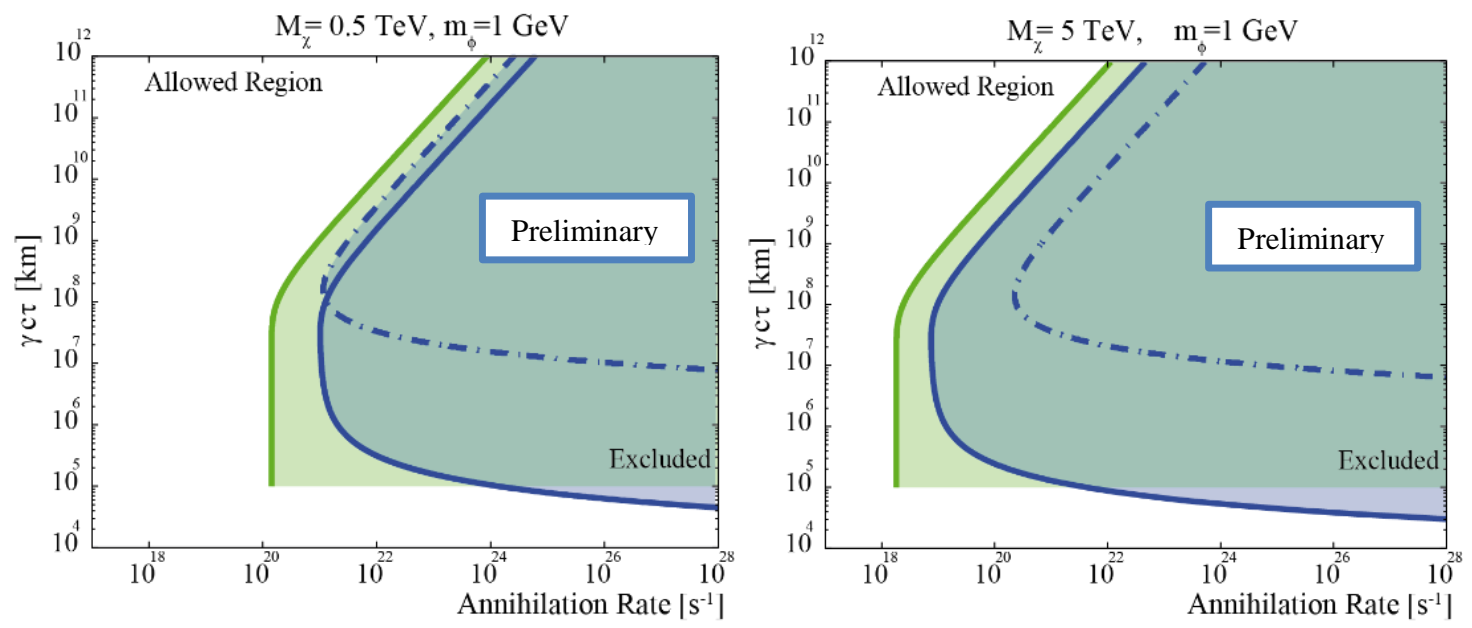

Figure 2. ANTARES exclusion limits for the Secluded DM cases studied (products of DM annihilation in the Sun through mediators decaying into: di-muons (dash-dotted blue), neutrinos from di-muons (solid blue), directly into neutrinos (Green)) as a function of the annihilation rate $(\Gamma)$ and the decay length $(\gamma \mathrm{c} \tau)$ for 0.5 and $5 \mathrm{TeV}$ DM masses. The shadow regions are excluded for these models. 
Limits on DM-nucleon interaction can also be derived for these cases. Assuming equilibrium of the DM population in the Sun, i. e., the annihilation balances the DM, $\Gamma=C_{D M} / 2$, and according to [25] the capture is approximately:

$$
C_{D M}=10^{20} s^{-1}\left(\frac{1 T e V}{M_{D M}}\right)^{2} \frac{2.77 \sigma_{S D}+4270 \sigma_{S I}}{10^{-40} \mathrm{~cm}^{2}}
$$

where, $\sigma_{\mathrm{SD}}$ and $\sigma_{\mathrm{SI}}$ are the spin-dependent (SD) and spin-independent (SI) cross-sections, respectively, and $\mathrm{M}_{\mathrm{DM}}$ is the DM mass. The limits on the SD and SI WIMP-proton scattering cross-sections are derived for the case in which one or the other is dominant. The sensitivity in terms of the annihilation rates depends on the lifetime of the mediator. To assess the potential to constrain these models, lifetime values for which the sensitivities are the best possible have been assumed. For the di-muon case, the lifetime has to be long enough to assure that the mediator reaches the vicinity of the Earth, so mediators with decay length about Sun-Earth distance are shown. In both neutrino cases the lifetime of the mediator for best sensitivity has to be long enough to ensure that the mediator escapes the Sun, but not too long so that it decays before reaching the Earth. The lifetime of the mediator for the best sensitivity has been chosen, corresponding to a distance of approximately forty times the solar radius. Figure 3 shows the ANTARES nucleon-WIMP cross-section limits for the SDM scenario (products of DM annihilation in the Sun through mediators decaying into: di-muons (blue) and directly into neutrinos (green)) for the selected mediator's lifetimes. The limits are compared to those given by different experiments of direct search for dark matter.
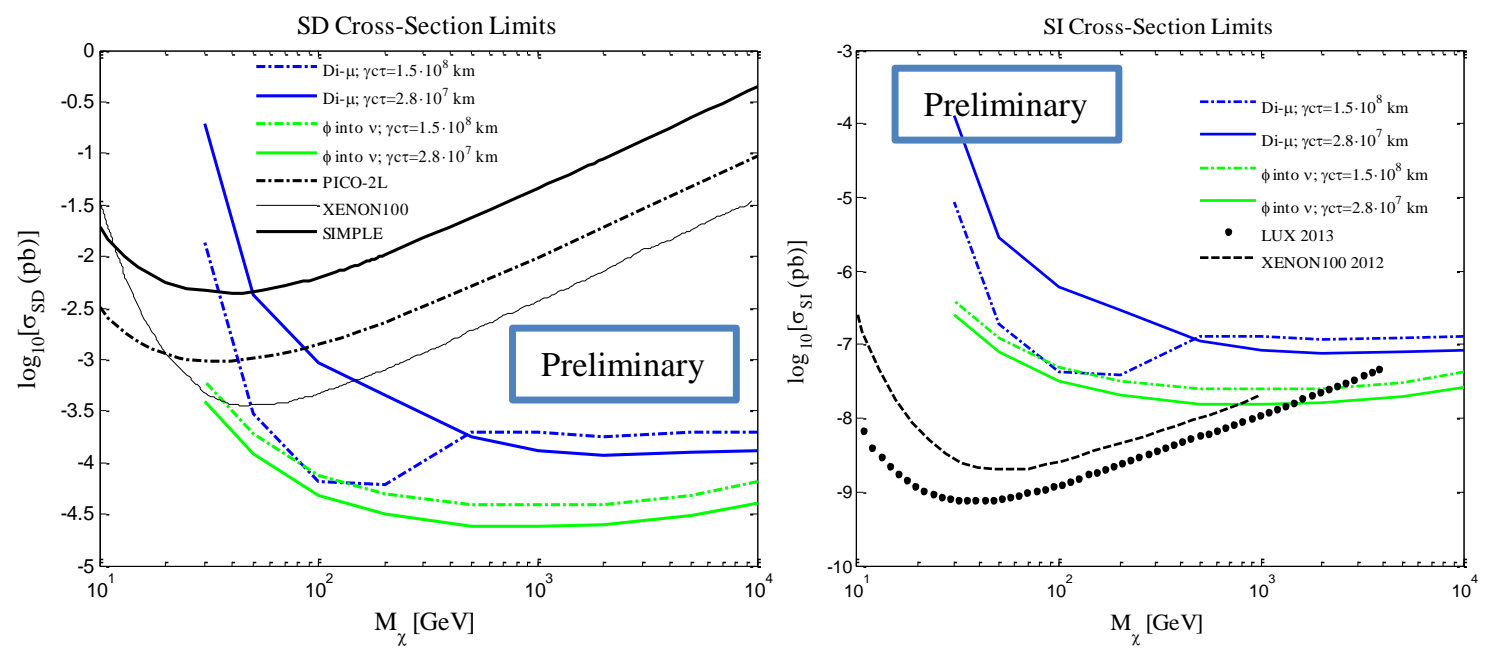

Figure 3. ANTARES 90\% CL upper limits on WIMP-nucleon cross-section as a function of WIMP mass. The left panel refers to spin-dependent and the right one to spin-independent WIMPs interactions. Two favourable mediator lifetimes are considered. The procedure to translate from DM annihilation rate in the Sun, $\Gamma$, and $\mathrm{c} \tau$ to $\mathrm{M}_{\chi}$ and $\sigma_{\chi}$ is described in [15]. Additionally, in the same panel the current bounds from SIMPLE [26], PICO [27], LUX [28] and XENON [29] are plotted.

The limits derived here are the first experimental limits on SDM models established by neutrino telescopes. There were some previous constrains or sensitivities predicted by phenomenology physicists [14,15], but naturally, the knowledge of the response of the detector in this kind of studies is quite limited, and therefore, the results should be taken with caution. As shown in Figure 3, for sufficiently long-lived, but unstable mediators, the limits imposed to 
these models are much more restrictive than those derived in direct detection searches for the case of spin-dependent interaction. In the case of spin-independent interactions, direct detection search is more competitive for low and intermediate masses, but the SDM search becomes more competitive for larger masses $(>1 \mathrm{TeV})$.

Compared to other indirect detection methods, such as those using gamma-rays, the limits derived here are in general competitive for large dark matter masses and favourable mediator lifetimes $\left(\gamma c \tau \sim 10^{11} \mathrm{~m}\right)$. However, the comparison is not straightforward, since the results are usually given in terms of the $\langle\sigma v\rangle$ parameter and several astrophysical assumptions have to be made. Therefore, the different indirect searches can be considered to provide complementary information. In that sense, this analysis constrains in an alternative way these models that are one of the preferred solutions to explain, for example, the energy of the positron flux measured by AMS-II [12]. Although one possible interpretation of this data would be the existence of nearby pulsars, a great deal of papers study the possibility of a DM hint. In this line, the annihilation into two mediators that results in four leptons (two di-muons, for example) is more favoured than the direct annihilation into leptons [30-32].

\section{Acknowledgements}

We acknowledge the financial support of the Spanish Ministerio de Economía y Competitividad (MINECO) and Ministerio de Ciencia e Innovación (MICINN), Grants FPA2012-37528-C02-02, and Consolider MultiDark CSD2009-00064, and of the Generalitat Valenciana, Grant PrometeoII/2014/079.

\section{References}

[1] S. Adrián-Martínez et al. JCAP 2013, 032 (2013).

[2] M. M. Boliev et al. JCAP 1309, 019 (2013).

[3] T. Tanaka et al. Astrophys. J 742, 78 (2011).

[4] M. G. Aartsen et al. Phys. Rev. Lett. 110, 131302 (2013) .

[5] M. Pospelov, A. Ritz, M. B. Voloshin. Phys. Lett. B662, 53 (2008).

[6] N. Arkani-Hamed, D. P. Finkbeiner, T. R. Slatyer, N. Weiner. Phys. Rev. D79, 015014 (2009).

[7] M. Pospelov, A. Ritz. Phys. Lett. B671, 391 (2009).

[8] I.Z. Rothstein, T. Schwetz and J. Zupan JCAP 07, 18 (2009).

[9] F. Chen, J. M. Cline, A. R. Frey. Phys. Rev. D80, 083516 (2009).

[10] O. Adriani et al. Phys. Rev. Lett. 105, 121101 (2010).

[11] A.A. Abdo et al.. Phys. Rev. Lett. 102, 181101 (2009).

[12] L. Accardo, et al., Phys. Rev. Lett., 113, 121101 (2014). M. Aguilar, et al., Phys. Rev. Lett., 113, 121102 (2014).

[13] B. Batell, M. Pospelov, A. Ritz, Y. Shang. Phys. Rev. D81, 075004 (2010).

[14] P. Schuster, N. Toro, N. Weiner, I. Yavin. Phys. Rev. D82, 115012 (2010). 
[15] P. Meade, S. Nussinov, M. Papucci, T. Volansky. J. High Energy Phys. 06 2010:29 (2010)

[16] J. Miller et al., Search for secluded DM in the Sun with IceCube, Exotic Physics with Neutrino Telescopes Workshop, 2013.

[17] N. F. Bell, K. Petraki. JCAP 2011, 003 (2011).

[18] M. Ageron, et al., Nucl. Instrum. and Meth. A 656, 11 (2011).

[19] S.Adrián-Martínez et al. J. Instrum. 7, T08002 (2012). M. Ardid, for the ANTARES collaboration, Nucl. Instrum. and Meth. A 602, 174 (2009).

[20] J.A. Aguilar et al., Astropart. Phys. 34, 539 (2011).

[21] J.A. Aguilar et al., Astropart. Phys. 34, 652 (2011).

[22] S. Adrián-Martínez, Ph. D. Thesis. Universitat Politècnica de València (2015).

[23] G. C. Hill, K. Rawlins. Astropart. Phys. 19, 393 (2003).

[24] G. J. Feldman, R. D. Cousins. Phys.Rev.D. 57, 3873-3889 (1998).

[25] A. Ibarra, M. Totzauer, S. Wild. JCAP. 1404, 012 (2014).

[26] M. Felizardo et al. Phys. Rev. Lett. 108, 201302 (2012).

[27] C. Amole et al. Phys. Rev. Lett. 114, 231302 (2015).

[28] D. S. Akerib et al., Phys. Rev. Lett., 112, 091303 (2014).

[29] E. Aprile et al., Phys. Rev. Lett., 109, 181301 (2012).

[30] I. Cholis, D. Hooper, Phys. Rev. D 88, 023013 (2013).

[31] M. Boudaud et al, A\&A 575, A67 (2015).

[32] A. Lopez et al., arXiv:1501.01618 (2015). 